\title{
Off-line Handwritten Bilingual Name Recognition for Student Identification in an Automated Assessment System
}

\author{
Hemmaphan Suwanwiwat, Vu Nguyen, and \\ Michael Blumenstein \\ School of Information and Communication Technology, \\ Griffith University, Australia
}

\begin{abstract}
The Student name Identification System (SIS) proposed here was investigated for English and Thai languages combined. The proposed system recognises each name by using an approach for whole word recognition. In the proposed system, the Gaussian Grid Feature (GGF), and Modified Direction Feature (MDF), together with a proposed hybrid feature extraction technique called Water Reservoir, Loop and Gaussian Grid Feature (WRLGGF) were investigated on full word contour images of each name sample; Artificial neural networks and support vector machines were used as classifiers. An encouraging recognition accuracy of $99.25 \%$ was achieved employing the proposed technique compared to $98.59 \%$ for GGF, and 96.63\% using MDF.
\end{abstract}

Keywords-Off-line handwriting recognition; Automated assessment system; Bilingual Student Identification System; Water reservoir feature; Gaussian grid feature

\section{INTRODUCTION}

Off-line Automatic Assessment Systems (OFAAS) and Student Identification Systems (SIS) benefit from off-line handwriting recognition techniques. There is not much work that has been reported on SIS [1]. In addition, there is only a small amount of research focusing on OFAAS [1], [2], [3], [4]. Commonly for manual assessment, when the marking of examination papers is concluded, the marker has to rewrite each student's mark onto a marking summary sheet. One problem of transcribing the mark of each student is that it could be error prone as the assessor may mistakenly ascribe the examination mark against the wrong student name.

This paper proposes a sub-system of an OFAAS called the Bilingual Student Identification System (BSIS), the two languages being Thai and English. The BSIS was created based on the fact that in some Thai examination papers, both Thai and English student names (written their names in Thai and English languages), together with the student number were written down to identify each student. For the BSIS, once the marking process is completed, the report on each student's mark is automatically produced. Having such a system would reduce the chance of mismatching the student's names and their marks.

The BSIS was employed on the Thai and English languages to identify students' first and last names (name components). To the best of the authors' knowledge, there is no off-line bilingual automated assessment systems proposed in the literature. Also, there has not been much work done on

\author{
Umapada Pal \\ Computer Vision and Pattern Recognition Unit \\ Indian Statistical Institute \\ India
}

Thai whole word recognition, and especially not on writer identification as per the work that is proposed here. This is due to the nature of the Thai language (see Section II-B).

BSIS recognises Thai words as well as English words; however, Thai words have different characteristics when compared to English words. In order to develop a BSIS that efficiently recognises both languages, a new feature extraction technique has been applied to the system.

A novel hybrid feature extraction technique called Water Reservoir, Loop and Gaussian Grid Feature (WRLGGF), together with the Modified Direction Feature (MDF), and Gaussian Grid Feature (GGF) were employed in a performance investigation and comparison. Artificial Neural Networks (ANNs) and Support Vector Machines (SVMs) were used as classifiers to compare the recognition rates on the techniques.

As there is no suitable dataset of Thai name components available, a new dataset was created to be used for experimentation of the proposed system. Altogether, the dataset used in the present research consists of 4,120 handwritten name components from a total number of 206 writers when combined with the English name component dataset (further details are discussed in Section II-A).

The remainder of this paper is organised into three sections. Section II describes the methodology employed in this research. Section III details the results obtained and puts forward a discussion and analysis. Finally, conclusions are drawn in Section IV and future work is also described.

\section{METHODOLOGY}

This section discusses the methodology and techniques used in conducting the research. The topics in this section include the proposed system (block diagrams), data collection, nature of the Thai language, proposed methodology, and the experimental setup.

The proposed methodology includes data collection, image processing, and the investigation of the WRLGGF, MDF, and GGF features in conjunction with classifiers employing different parameters in order to achieve the optimum results. Classifiers used in conducting the proposed BSIS experiments are ANNs and SVMs. Fig. 1a illustrates a block diagram of a complete OFAAS, which consists of a main component and this is composed of a short answer question automatic marking module, and a BSIS, which is a sub-system of OFAAS. 


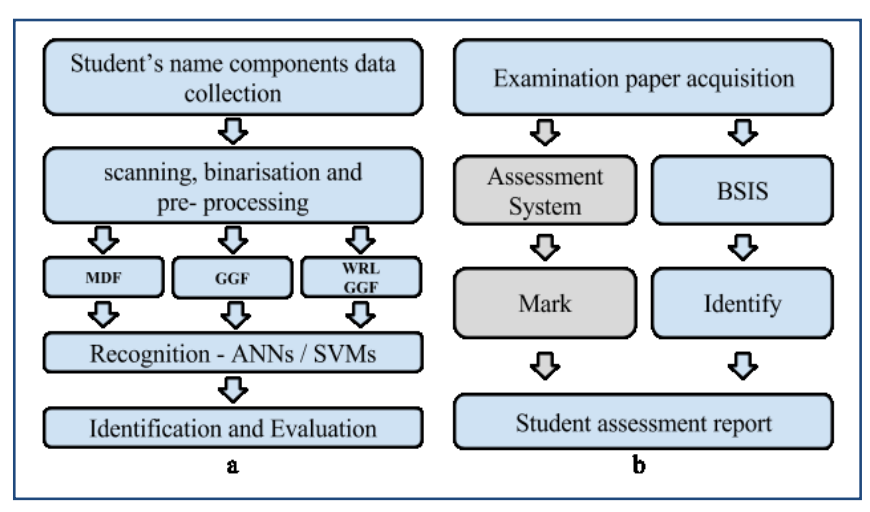

Figure 1a. A block diagram illustrating a complete Off-Line Automatic Assessment System (OFAAS). Figure 1b. A block diagram illustrating the proposed Bilingual Student Identification System (BSIS).

BSIS identifies students from their name components (first and last name). Once both processes are completed, the full report containing a list of students who attended the examination along with the marks they achieved is produced. The BSIS process begins with the data collection of the students' name components. The scanning process is used to transform raw data into digitised patterns. Binarisation and preprocessing, including line and word segmentation, noise removal, filling and skew correction are then applied to the images.

The feature extraction techniques which were selected for investigation are the newly proposed WRLGGF, MDF and GGF. After the feature vectors are generated by applying each technique to the relevant digital images, the features are then input to the ANNs and SVMs for training, and testing for the recognition/identification process. The BSIS recognition accuracy rate was evaluated once the results were obtained. A proposed BSIS block diagram can be found in Fig. 1b.

\section{A. Data Collection}

There has been no publicly available dataset of Thai language handwritten name components from examination papers; as a result, a data collection process was performed to create a custom dataset. The dataset collected for the proposed system is the first of its type in the Thai language. In the research proposed here, the recognition of words was based on one writer per name components. Upon request, the dataset is available for download to the research community.

\begin{tabular}{|c|c|c|}
\hline Intra-class & Tधकरण口? & 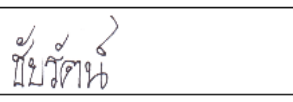 \\
\hline Intra-class & Sinemuinet & Sunanwinat \\
\hline Inter-class & Jooywattana & Jooywattana \\
\hline Inter-class & & \\
\hline
\end{tabular}

Figure 2. Name component examples. Intra-class differences, various lengths of name components, and duplication of name components written by different writers can be found.
One hundred and three volunteers, both male and female, provided handwritten Thai language first and last names in order to be used for training and testing the proposed BSIS. In total, there were 2,060 (206 name components $\times 10$ samples of each name component) samples obtained. This Thai dataset was subsequently combined with the English dataset.

The English name component dataset was obtained from the authors' previous work [1] plus 6 additional English name components from 3 writers. To sum up, the bilingual dataset was increased to 4,120 (412 name components $\times 10$ samples of each name component) samples. All samples were written with minimum constraints (e.g. writing instruments and handwriting styles were not restricted within the given space). Since the Thai name component dataset was collected at a different time to the English name component dataset, most the writers were not from the same group of people and therefore most of Thai and English names are not matched (did not belong to the same person).

Each volunteer wrote their name components one after another, ten times. Even though the volunteer wrote their name components one after the other, intra-class variations were observed (Fig. 2). Some of the name components are particularly long, for example, สวรรค์บรรจบริการ, and Chalermwutthiphong.

For the Thai language, one of the name characteristics is that the name components may begin with, end, or include some common words for name components such as "wat", "chai", "ya", "kit" (วัฒน์, ชัย, ยา, กิต, respectively), and therefore can be quite confusing for automatic classification because of the common characteristics, which were shared by the volunteers. In summary, the dataset contains names with quite varied word lengths (from 2-7 syllables which can be up to 18 characters), some duplicated last names, and some of the name components containing common words within each component. This is illustrated in Fig. 2. Intra-class differences were found and name component duplication of different writers existed (the name components of "Jooywattana" were written by two different writers).

\section{B. Thai Language}

The Thai language is considerably different from the English language. It consists of 44 consonants, 18 vowels, 4 voice tones, and 3 special symbols. Excluding Thai numerals, there are up to 69 characters in the Thai language. Heads of Thai characters, which are small loops, play an important role because many of the characters look the same. Only the position or whether or not the head is present will identify what character it is.

The heads of a character can be found in various positions. An obvious example that shows the importance of the head is these three different characters that are only differentiated because of their heads: "ก", "ภ" and "ถ". Loops (heads of characters) can be found in many positions, including upper-left part of a character ("У"), upper-right part of a character (“ห”), middle part of a character (“ø.), lower left part of a character (“"”), lower right part of character (“น"). 
There is no space between words for the Thai language, which makes it hard to segment words from a sentence. For example "ตากลม" can mean sitting in the wind (ตาก ลม) or sitting with your eyes wide open (ตากลม). However this is not a problem for the research proposed here as the names were recognised as a whole (whole word recognition).

\section{Student Name Component Dataset}

For WRLGGF, GGF, and MDF extraction techniques, a total number of 4,120 word samples were used for classifier training and testing. $80 \%$ of the samples of each name were used as the training set, and $20 \%$ were used as the testing set. Altogether there are $412 \times 8=3,296$ samples used for training and $412 \times 2=824$ samples used for testing. Ten-fold cross validation was employed across all 4,120 samples for classification.

\section{Image Acquisition and Preprocessing}

Image preprocessing is a necessary operation which needs to be performed before the recognition process. The main purpose of image preprocessing is to develop useful canonical descriptions of shapes and surfaces in the given image [5]. Outputs from these preprocessing steps are used as input for the training and recognition phase; hence, some preprocessing techniques were applied in order to achieve the most valid output.

A scanner with 300 dpi resolution was used to perform image acquisition. The scanned images were stored in a grey-level format. After that, Otsu's thresholding algorithm was performed in order to obtain a binarised image [6]. Automatic line and word segmentation were performed using histogram projection. Line segmentation was performed first, and then word segmentation was performed in order to obtain each of the name components (first and last name). Salt and pepper noise removal and filling techniques were also applied. Skew normalisation was performed on each name image. Slant correction was not performed on any of the images to preserve the unique characteristics of each student name.

For each image, boundary extraction was performed to isolate connected components. A boundary extraction algorithm was employed [7]. After that, loop as well as upper and lower contour extraction was performed. It is to be noted

\begin{tabular}{|ll|}
\hline Original grey-level image & $6902 \mathrm{Hem}$ \\
Binarised image & $692 \mathrm{Hem}$ \\
After skew normalis ation & $6902 \mathrm{Hem}$ \\
After applying noise removal, filling, & 6 \\
and boundary extraction & \\
After loop extraction & \\
After upper contour extraction & \\
After lower contour extraction &
\end{tabular}

Figure 3. Example images obtained after each preprocessing step. that upper and lower contour extraction was performed in order to find upper and lower reservoirs only and not to be used as features themselves, therefore a skeletonisation approach was not considered in this research. Examples of preprocessing successive images are presented in Fig. 3.

\section{E. Feature Extraction}

Feature extraction is one of the most crucial aspects of a handwriting recognition system. The objective of feature extraction is to extract the salient information that needs to be applied in the recognition process. It reduces data dimensionality by determining certain feature properties that distinguish input patterns [8]. A feature extraction technique called WRLGGF is proposed here. Also MDF and GGF extraction techniques were chosen due to their ability to successfully extract important features from images, which have enabled accurate recognition rates to be attained in a number of applications [3], [9].

Feature vector sizes of the three techniques are quite different; the WRLGGF vector size is $1,448,121$ for MDF, and 864 for GGF. It is noted that the three feature extraction techniques were applied separately and were not combined in any way. Five features that were implemented in this research being MDF, GGF, water reservoir features, loop features, and the proposed WRLGGF are explained here.

1) The Modified Direction Feature (MDF) Extraction Technique: Originally, the MDF was created to extract direction and location information at background to foreground transitions from handwritten characters. However, in the proposed system, the MDF will be implemented to extract information from the whole word (each name component) image. Likewise, the recognition process will utilise holistic name information rather than recognising the handwriting at the character level.

MDF builds upon the Direction Feature [10]. MDF feature vector creation is based on the calculation of transition features from background to foreground pixels in the vertical and horizontal directions. The vector size is 121 when applying MDF to the full boundary image.

2) The Gaussian Grid Feature (GGF) Extraction Technique: The Gaussian Grid Feature [9] is a relatively new feature extraction technique. Originally, it was developed for the signature verification problem. The GGF employs pattern contours as its input. From the contour representation of a name component image, the contour image is divided into $12 \times 12$ zones of equal size. By tracing the contours in each block, the 4-direction chain code histogram of each block is created. A Gaussian smoothing filter $(\sigma=1.2)$ is then applied to each directional $12 \times 12$ matrix. The value of each element of each matrix obtained in the previous step is then normalised by dividing its value by the maximum value of the four matrices. From the two-matrix pairs, horizontal $(\mathrm{H})$ and vertical (V) matrices, left-diagonal (L) and rightdiagonal $(\mathrm{R})$ matrices, two new matrices $(\oplus$ and $\otimes)$ are established by manipulating pairs of matrices of the perpendicular directions. The feature vector is formed by merging the six matrices $H, V, L, R, \oplus$ and $\otimes$. The dimension of the output feature vector is $12 \times 12 \times 6=864$. 
3) Water Reservoir Feature (WRF) Extraction Technique: Generally, the Water Reservoir (WR) feature was used in segmentation tasks in both numeric and word segmentation [11], [12]. However, in the research proposed here, the technique was used to locate WRs found in upper and lower contours of images (refer to Fig. 4). After the reservoirs are found, the features of these reservoirs are extracted. WR features were used in the research proposed here because both Thai and English have this property in their scripts (see Fig. 4). WR properties can be seen in Fig. 5.

The WR principle supposes that if water is poured from the side of a script component, the cavity regions of the background portion of the component where water will be stored are considered as reservoirs of the component. To find water reservoirs, first upper and lower contours are found. The top reservoir (located at the upper contour) is found if the water is poured from the top of the component, and the bottom reservoir (located at the lower contour) is found where the water is poured if rotating the component by $180^{\circ}$.

For each contour image, the image was divided into seven equally-sized windows. In each window, once each WR was located (if any), seven WR features were extracted. The seven WR features used in this research are:

- WR area which is the area of the cavity region where water will be stored. The number of pixels inside a reservoir is tallied and is considered the area (1 feature).

- WR level that overflows from a reservoir (1 feature).

- WR flow side is where the water flows from; this can be left, right or either side, if the flow level is the same for both sides ( 2 features).

- $\quad$ WR base line (Y co-ordinate) (1 feature).

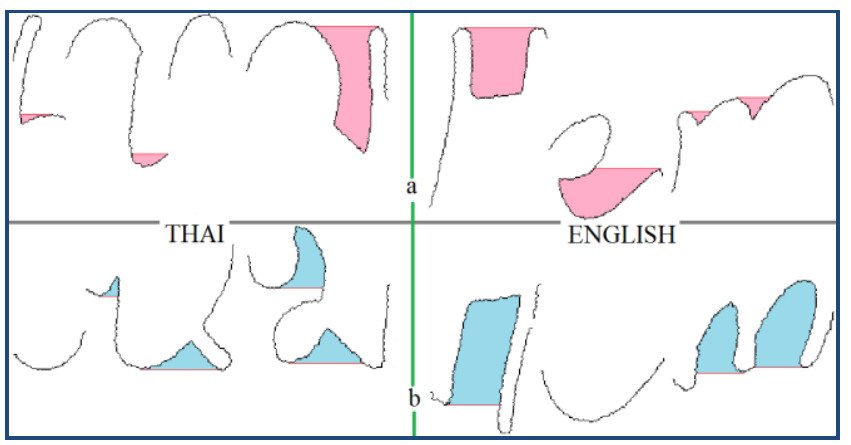

Figure 4. (a) Top WRs retrieved from the upper contour, and (b) bottom WRs retrieved from the lower contour. The full contour image can be seen in Fig 3.

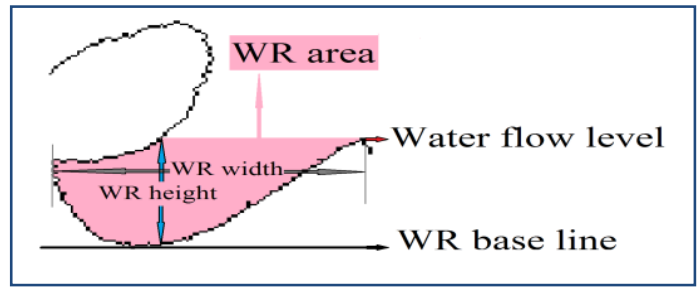

Figure 5. Water reservoir properties.
- WR width and height (2 features).

The ratio between width and height was applied to each feature as none of the images were size normalised. The number of WRs is set to four per window based on the assumption that there are less than 4 WRs per window. This makes the WR feature vector 169 ( 7 windows $\times 4$ WRs $\times 7$ features) in size per contour image; and so makes the vector size equal to 392 for both upper and lower contour images.

4) Loop Feature (LF) Extraction Technique: As the nature of both Thai and English languages may contain loops in each character and because for the Thai language, the loops play an important role in distinguishing characters (refer to Section B), the research proposed here also extracts features from loops found in images (please refer to Fig. 3).

In order to obtain loop features, loop images are first divided into 3 zones (baseline, middle, and top zone). Then loops were located within each zone. Each zone allows fifteen loops to be stored in a feature vector. Each loop has four features being the loop area, which is obtained from the total number of pixels inside a loop, loop height, loop width and the zone that the loop is located in. Therefore, for the dataset investigated, the loop feature vector size is 192; the total number of 192 is comprised of 3 zones $\times 15$ loops from each zone $\times 4$ features of each loop +12 additional features for the average loop area, average loop width and height, and total number of loops of each zone.

5) Water Reservoir, Loop, and Gaussian Grid Feature (WRLGGF) Extraction Technique: The proposed WRLGGF was developed based on three feature extraction techniques being WRF, LF and GGF. WRF and LF can be found in both Thai and English, and are important character identifiers for the Thai language. WRF and LF were combined with GGF in order to extract the most salient information, so that the best recognition rates possible can be achieved.

From full contour images, the process of top contour, bottom contour, and loop finding was undertaken. From full boundary images, there are three that were retrieved, being top contour image, bottom contour image, and loop images (see Fig. 3). For the top and bottom contour, each reservoir was located, then reservoir feature extraction (described in E.3) was applied on each reservoir and recorded in a feature vector. Once top and bottom reservoir extraction processes were completed, loop feature extraction (explained in E.4) was processed and features retrieved from each loop were recorded in the vector. Finally, GGF was then applied on the full boundary contour to extract features from the image. The final WRLGGF vector, size of 1,448 (392 from WRF + 192 from $\mathrm{LF}+864$ from GGF), was retrieved and ready for input into the classification process.

It should be noted that two additional feature extraction techniques, which are LF combined with GGF (LGGF), and WRF combined with GGF (WRGGF), were also used in the experiments for recognition rate comparison purposes.

\section{F. Classification}

The ANNs were trained with the resilient backpropagation algorithm, which was selected above all others to address the problem of magnitudes of the partial 
derivative effects when using the sigmoid function (details about ANN settings can be found in sub-section G). For both MDF and GGF features, the neural networks were trained using $412 \times 8=3,296$ samples, and tested using $412 \times 2=$ 824 samples. For the experiments using SVM, libsvm [13] was employed in conjunction with the WEKA toolkit [14]. For training the SVMs, ten-fold cross validation was used across all 4,120 handwriting samples.

\section{G. Experimental Settings}

For both ANN and SVM settings and structures, there were 412 outputs for the 412 first and last names. The duplicated name components from different writers, for example "สุวรรณวิวัฒน์" from "เหมพรรณ สุวรรณวิวัฒน์" and "สุวรรณ วิวัฒน์" from "บุญส่ง สุวรรณวิวัฒน์" were classified into 2 different outputs. However, in the recognition phase, "สุวรรณวิวัฒน์" can be recognised as either "สุวรรณวิวัฒน์" of เหมพรรณ's output or of บุญู่ง's output. The BSIS will identify who the name component belongs to. This is applied to both languages. Classification into 2 different outputs was carried out because in the future it is believed that this will be useful in developing the BSIS that can identify and verify students from their name components.

For ANNs, the number of hidden units investigated was experimentally set from 30 up to 130 hidden units, incrementing by 1 at a time. The number of iterations set for training increased from 1000 up to 20000, incrementing by 1000 at a time. All ANNs were trained with Matlab 2011a default parameters for resilient backpropagation. For the SVM experimental settings, ten-fold cross validation was performed to get statistically meaningful results. The Gaussian kernel was used and the C parameter of the SVM was set to be 100 .

\section{H. BSIS Evaluations}

Classification accuracy rates were evaluated by using the unseen testing dataset before being applied to the BSIS. The unseen testing dataset included 824 samples $(20 \%$ of the 4,120 samples). Only the best classification outcomes using each feature extraction technique from both classifiers (ANN and SVM) were applied to the proposed system (also see Section III for more details).

\section{EXPERIMENTAL RESULTS AND DISCUSSION}

This section presents recognition rates obtained from the ANN and SVM classifiers trained using MDF, GGF, WRGGF, LGGF or WRLGGF feature extraction techniques. ANNs and SVMs were trained using the five extraction techniques individually, and tested. The best recognition rates, together with their settings, are presented in Table I.

The best result of $99.25 \%$ was obtained using the WRLGGF in conjunction with SVMs. This result is $0.7 \%$ better than the GGF feature. The reason that the result is better when WR and LF were extracted from the full boundary (contour) images is that more important information was obtained and therefore assisted in the recognition process.
TABLE I. RECOGNITION RATES ATTAINED EMPLOYING MDF, GGF, WRGGF, LGGF OR WRLGGF FEATURE EXTRACTION TECHNIQUES IN CONJUNCTION WITH THE ARTIFICIAL NEURAL NETWORK (ANN) AND SUPPORT VECTOR MACHINE (SVM) CLASSIFIERS

\begin{tabular}{|c|c|c|c|c|c|}
\hline \multirow[b]{2}{*}{ Classifier } & \multicolumn{5}{|c|}{ Feature Extraction Techniques } \\
\hline & MDF & GGF & $\begin{array}{c}\text { WR } \\
\text { GGF }\end{array}$ & $\begin{array}{c}\mathbf{L} \\
\text { GGF }\end{array}$ & $\begin{array}{l}\text { WRL } \\
\text { GGF }\end{array}$ \\
\hline ANN recognition rate (\%) & 84.83 & 69.66 & 63.95 & 68.45 & 78.15 \\
\hline Hidden units & 127 & 120 & 111 & 97 & 121 \\
\hline Iterations & 2000 & 3000 & 2000 & 4000 & 2000 \\
\hline SVM recognition rate (\%) & 96.63 & 98.59 & 99.17 & 98.81 & $\underline{99.25}$ \\
\hline
\end{tabular}

The highest recognition rate obtained when applying MDF, which is $96.63 \%$, did not outperform the recognition rates attained using WRLGGF (99.25\%), WRGGF (99.17\%), LGGF (98.81\%), nor the original GGF which obtained $98.59 \%$ (all best rates were obtained using SVMs).

For all feature extraction techniques, the training times required when applying the MDF were shorter than WRLGGF, WRGGF, LGGF, and GGF due to its smaller feature vector size. Also as can be seen, better recognition rates were obtained when using SVMs as the classifier. After each of the name components were recognised, the BSIS was used to map them against each student's name components. In order for the system to identify the student whom the name components belong to, it employs the following criteria (refer to Fig. 6).

When comparing the recognition rates of classifiers employing WRLGGF, GGF (MDF is excluded due to the poorer recognition rates received) extraction techniques and the identification accuracy of the BSIS with the human marker together with the above criteria, a $100 \%$ accuracy was attained. The $100 \%$ accuracy was obtained, mainly because of the efficiency of the feature extraction techniques used, combined with an ability of the BSIS and its criteria to identify who the name components belong to, and its ability to reject some name components for manual identification (see Fig. 6). In addition, both Thai and English names are available for each student name identification, even if some of the name components are misrecognised, the other name

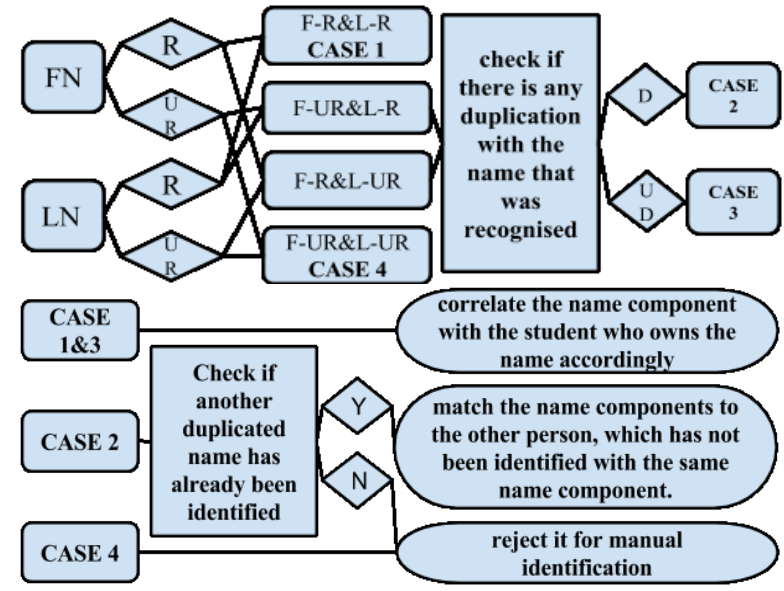

Figure 6. BSIS criteria decisions based on whether or not first (FN) or last (LN) name components are recognised (R) or unrecognised (UR), and if just one of the components is recognised, or whether there is any duplication (D) of first or last names 
TABLE II. COMPARISON BETWEEN THE RESUlts OF THE PROPOSED BSIS SYSTEM AND PREVIOUS WORK [3]

\begin{tabular}{|l|c|c|}
\hline \multicolumn{1}{|c|}{ System - Technique - Classifier } & RR (\%) & Database Size \\
\hline English SIS - MDF - ANN [1] & 98.59 & 2,040 words \\
\hline English SIS - GGF - ANN [1] & 93.62 & 2,040 words \\
\hline English SIS - GGF - SVM & 99.55 & 2,040 words \\
\hline English SIS - WRLGGF - SVM & $\mathbf{9 9 . 6 1}$ & $\mathbf{2 , 0 4 0}$ words \\
\hline English SIS - WRGGF - SVM & 99.56 & 2,040 words \\
\hline English SIS - LGGF - SVM & 99.41 & 2,040 words \\
\hline BSIS - WRLGGF - SVM & $\mathbf{9 9 . 2 5}$ & $\mathbf{4 , 1 2 0}$ words \\
\hline
\end{tabular}

components of the other language are still available for identification. It should be noted that a name component is classified as correctly Recognised (R) when it has the highest recognition rate from the classifier matching with the corresponding correct name of that name component; An Unrecognised (UR) name component is classified vice versa.

A related work found in the literature was performed in the context of automated assessment in the English language [1] with a different dataset size of 2,040 words. To be able to compare recognition rates attained from the proposed WRLGGF, more experiments were carried out by using the proposed WRLGGF together with WRGGF, LGGF and GGF techniques using the SVM on the English dataset of 2,040 words [1]. Recognition rates of the proposed WRLGGF together with a comparison of the existing system [1] and the proposed system are displayed in Table II.

As can be seen, when using WRLGGF on the English SIS dataset [1], and applying it to SVMs, a higher recognition rate of $99.61 \%$ was obtained compared to $98.59 \%$ for MDF, $93.62 \%$ for GGF using ANNs, and $99.55 \%$ of the original GGF using SVMs. It can be noted that even though the BSIS has a larger and more complex dataset (bilingual), the recognition rate achieved is comparable to the English SIS $(99.25 \%$ for the BSIS compared to $99.61 \%$ for the English SIS respectively).

\section{CONCLUSIONS AND FUTURE RESEARCH}

This paper deals with a SIS for the bilingual scenario (BSIS). There is no work on BSIS in the literature; hence such work will be useful for the research community. This research presented a comparative performance analysis of five distinct feature extraction techniques within the context of a whole-word recognition system, which is proposed in the paper. Two of which (GGF and MDF) were originally designed for different purposes, character recognition and signature verification respectively, rather than handwritten whole-word recognition. The experimental results obtained in this research indicate that the performance of one of the proposed techniques is encouraging and comparable. The highest accuracy obtained by applying the proposed WRLGGF technique is $99.25 \%$ which is comparable to that which was obtained when the MDF extraction technique was employed (96.63\%).

It is of interest to find that the newly proposed WRLGGF combination yielded a better recognition rate when comparing it to the original GGF. The additional WR and LF complemented GGF efficiently; better recognition rates were obtained for both SVMs and ANNs (from 69.66\% to 78.15\% for ANNs and from $98.59 \%$ to $99.25 \%$ for SVMs). However, more experiments will need to be carried out on a larger dataset with other scripts to observe the results. More languages may be investigated in the future to find out if the proposed system is generic enough to support a broader range of languages. Also, as the WRLGGF feature vector is quite large, compression/feature selection techniques may be applied in future work. Furthermore, a hybrid MDF combining LF and WRF, will be investigated.

In addition, the work will be extended to student verification so that the system can detect if the students who sat an examination were really those who own the name and not someone else.

\section{REFERENCES}

[1] H. Suwanwiwat, V. Nguyen, M. Blumenstein. Off-line restricted-set handwritten word recognition for student identification in a short answer question automated assessment system, 12th International Conference on Hybrid Intelligent Systems (HIS 2012), pp.167-172, 2012

[2] J. Allan. Automated Assessment Of Handwritten Scripts. PhD thesis, Nottingham Trent University, 2004.

[3] H. Suwanwiwat and M. Blumenstein. Short answer question examination using an automatic off-line handwriting recognition system and the modified direction feature. In 3rd International Conference on Machine Version (ICMV 2010), pages 476-480, 2010.

[4] S. Srihari, J. Collins, R. Srihari, H. Srinivasan, S. Shetty, and J. BruttGriffler. Automatic scoring of short handwritten essays in reading comprehension tests. Artificial Intelligence, 172:300-324, 2008.

[5] A D. Kulkarni. Computer vision and fuzzy-neural systems. Prentice Hall PTR, Upper Saddle River, NJ, 2001.

[6] N. Otsu. A threshold selection method from gray level histograms. Systems, IEEE Transactions on Man and Cybernetics, 9(1):62-66, 1979.

[7] J. R. Parker, 1994, Practical Computer Vision using C, John Wiley and Sons, New York, NY.

[8] C.c. Tappert, c. y. Suen, T. Wakahara, "The State of the Art in Online Handwriting Recognition," IEEE Transactions on Pattern Analysis and Machine Intelligence, August 1990, pp. 787-808

[9] V. M. Nguyen and M. Blumenstein. An application of the 2D Gaussian filter for enhancing feature extraction in off-line signature verification. In $11^{\text {th }}$ International Conference on Document Analysis and Recognition (ICDAR 2011), pages 339-343, 2011.

[10] M. Blumenstein, X. Y. Liu, and B. Verma. A modified direction feature for cursive character recognition. In IntI. Joint Conference on Neural networks, volume 4, pages 2983-2987, 2004.

[11] U. Pal, A. Belaid and Ch. Choisy "Touching numeral segmentation suing water reservoir concept", Pattern Recognition Letters, vol.24, pp. 261-272, 2003.

[12] Jayadevan, R.; Pal, U.; Kimura, F., "Recognition of Words from Legal Amounts of Indian Bank Cheques," International Conference on Frontiers in Handwriting Recognition (ICFHR), pp.166-171, 2010.

[13] Chang, Chih-Chung and Lin, Chih-Jen, LIBSVM: A library for support vector Machines, ACM Transactions on Intelligent Systems and Technology, vol:2(3), 2011, pp.1-27

[14] Mark Hall, Eibe Frank, Geoffrey Holmes, Bernhard Pfahringer, Peter Reutemann, Ian H. Witten (2009); The WEKA Data Mining Software: An Update; SIGKDD Explorations, Volume 11, Issue 1. 